\title{
ON THE TRIANGULATION OF MANIFOLDS AND THE HAUPTVERMUTUNG
}

\author{
BY R. C. KIRBY ${ }^{1}$ AND L. C. SIEBENMANN ${ }^{2}$ \\ Communicated by William Browder, December 26, 1968
}

1. The first author's solution of the stable homeomorphism conjecture [5] leads naturally to a new method for deciding whether or not every topological manifold of high dimension supports a piecewise linear manifold structure (triangulation problem) that is essentially unique (Hauptvermutung) cf. Sullivan [14]. At this time a single obstacle remains ${ }^{3}$ - namely to decide whether the homotopy group $\pi_{3}(\mathrm{TOP} / \mathrm{PL})$ is 0 or $Z_{2}$. The positive results we obtain in spite of this obstacle are, in brief, these four: any (metrizable) topological manifold $M$ of dimension $\geqq 6$ is triangulable, i.e. homeomorphic to a piecewise linear $(=\mathrm{PL})$ manifold, provided $H^{4}\left(M ; Z_{2}\right)=0$; a homeomorphism $h: M_{1} \rightarrow M_{2}$ of PL manifolds of dimension $\geqq 6$ is isotopic to a PL homeomorphism provided $H^{3}\left(M ; Z_{2}\right)=0$; any compact topological manifold has the homotopy type of a finite complex (with no proviso); any (topological) homeomorphism of compact PL manifolds is a simple homotopy equivalence (again with no proviso).

$R$. Lashof and M. Rothenberg have proved some of the results of this paper, [9] and [10]. Our work is independent of [10]; on the other hand, Lashof's paper [9] was helpful to us in that it showed the relevance of Lees' immersion theorem [11] to our work and reinforced our suspicions that the Classification theorem below was correct.

We have divided our main result into a Classification theorem and a Structure theorem.

(I) Classification theorem. Let $M^{m}$ be any topological manifold of dimension $m \geqq 6$ (or $\geqq 5$ if the boundary $\partial M$ is empty). There is a natural one-to-one correspondence between isotopy classes of PL structures on $M$ and equivalence classes of stable reductions of the tangent microbundle $\tau(M)$ of $M$ to $P L$ microbundle.

(There are good relative versions of this classification. See [7] and proofs in §2.)

Explanations. Two PL structures $\Sigma$ and $\Sigma^{\prime}$ on $M$, each defined by a PL compatible atlas of charts, are said to be isotopic if there exists a

\footnotetext{
1 Partially supported by NSF Grant GP 6530.

2 Partially supported by NSF Grant GP 7952X.

${ }^{3}$ See note added in proof at end of article.
} 
topological isotopy $h_{t}, 0 \leqq t \leqq 1$, of $1_{M}$ so that $h_{1}$ is a PL homeomorphism of $(M, \Sigma)$ with $\left(M, \Sigma^{\prime}\right)$. If $M$ has a metric $d$ and $\epsilon$ is a continuous function $M \rightarrow(0, \infty)$, then $h_{t}$ is called an $\epsilon$-isotopy provided $d\left(x, h_{t}(x)\right)$ $<\epsilon(x)$ for all $x \in M$ and all $t \in[0,1]$. To (I) we can add: Isotopic $P L$ structures are $\epsilon$-isotopic for any $\epsilon$.

By $n$-microbundle one can, by the Kister-Mazur theorem, understand simply a locally-product bundle with fiber Euclidean $n$-space $R^{n}$, and zero-section. If $\xi$ is then a TOP (=topological) microbundle, over a locally finite simplicial complex $X$, with fiber $R^{n}$, a reduction of $\xi$ to PL microbundle is given by a triangulation of $\xi$ as a PL microbundle over $X$. Two such triangulations of $\xi$ give equivalent reductions if the identity of $\xi$ is bundle isotopic to a PL isomorphism from the one PL microbundle structure to the other. The notion of stable reduction differs in allowing addition of a trivial bundle at any moment. Since $M$ is not a priori triangulable, one should, to define reductions, first pull back $\tau(M)$ to a homotopy-equivalent simplicial complex. The total space of a normal microbundle of $M \cup\{$ collar on $\partial M\}$ in $R^{m+k}$ ( $k$ large) is convenient. This technicality obscures, but does not destroy, the pleasant properties of the notion of reduction.

If $\mathrm{TOP}_{m} / \mathrm{PL}_{m}$ is the fiber of the map, $\mathrm{BPL}_{m} \rightarrow \mathrm{BTOP}_{m}$ of classifying spaces for microbundles, define TOP/PL as the telescope of the sequence $\mathrm{TOP}_{1} / \mathrm{PL}_{1} \rightarrow \mathrm{TOP}_{2} / \mathrm{PL}_{2} \rightarrow \mathrm{TOP}_{3} / \mathrm{PL}_{3} \rightarrow \cdots$ arising from stabilization of bundles.

(II) Structure theorem (Partially announced iN [8]). $\pi_{k}(T O P / P L)$ is 0 if $i \neq 3$ and $Z_{2}$ or 0 if $i=3$. Also $\pi_{k}\left(T O P_{m} / P L_{m}\right)$ $\cong \pi_{k}(T O P / P L)$ by stabilization, for $k<m, m \geqq 5$.

When it became known that, with Wall, we had shown that $\pi_{k}$ (TOP/PL) is 0 for $k \neq 3$ and $\leqq Z_{2}$ for $k=3$, we [8] and Lashof and Rothenberg [10] independently noticed that Lees' immersion theorem [11] gives the corresponding nonstable results above. This was of critical importance to Lashof's triangulation theorem [9].

The equivalence classes of stable reductions of $\tau(M)$ can be put in one-to-one correspondence with vertical homotopy classes of sections of a bundle over $M$ with fiber TOP/PL, namely the pull-back by a classifying map $M \rightarrow B_{\text {TOP }}$ for $\tau(M)$ of the fibration TOP/PL $\rightarrow B_{\mathrm{PL}}$ $\rightarrow B_{\text {Top }}$. Combining (I) and (II) we find

(1) There is just one well-defined obstruction in $H^{4}\left(M ; \pi_{3}(T O P / P L)\right)$ to imposing a $P L$ structure on $M$.

(2) Given one $P L$ structure on $M$ the isotopy classes of $P L$ structures on $M$ are in (1-1)-correspondence with the elements of $H^{3}\left(M ; \pi_{3}(T O P / P L)\right)$. 
As applications of (I) alone consider:

(a) The total space $E$ of any normal $k$-disc bundle [3] of $M^{n}$ in $R^{n+k}, n+k \geqq 6$, is triangulable as a PL manifold, since $\tau(E)$ is trivial.

(b) If $h: E \rightarrow E^{\prime}$ is a homeomorphism of parallelizable PL $m$-manifolds, there exists a topological disc-bundle automorphism $\alpha: E \times D^{s}$ $\rightarrow E \times D^{s}$ over $E$ (s large) so that $(h \times i d) \circ \alpha: E \times D^{s} \rightarrow E^{\prime} \times D^{s}$ is topologically isotopic to a PL homeomorphism.

ProOF OF (b). The PL reduction of $\tau(E)$ given by $h$ is classified by an element $y \in\left[E, \mathrm{TOP}_{m} / \mathrm{PL}_{m}\right]$. Since $\tau(E)$ and $\tau\left(E^{\prime}\right)$ are trivial bundles, $y$ comes from $x \in\left[E\right.$, TOP $\left.{ }_{m}\right]$. Represent $-x$ by an automorphism $\beta: E \times R^{m} \rightarrow E \times R^{m}$ of the trivial $R^{m}$ bundle. Then, up to bundle isotopy $\beta \times 1_{R}$ extends [3] to a disc bundle automorphism $\alpha: E \times D^{m+1} \rightarrow E \times D^{m+1}$, where $R^{m+1}=\operatorname{int} D^{m+1}$. The PL reductions of $\tau\left(E \times D^{m+1}\right)$ given by $(h \times i d) \circ \alpha$ and by $i d \mid E \times D^{m+1}$ are stably the same; so (b) follows from (I).

These seemingly innocent observations readily affirm two important conjectures (cf. [13]).

(III) Finiteness of COMPACt TYPES. Every compact topological manifold has the homotopy type of a finite complex-even if it be nontriangulable.

(IV) Topological Invariance of torsions. Every topological manifold $M$ has a well-defined simple homotopy type $e^{4}$, namely the type of its normal disc bundles triangulated as PL manifolds. In particular, if $h: M \rightarrow M^{\prime}$ is a homeomorophism of compact connected PL manifolds, the Whitehead torsion $\tau(h) \in \mathrm{Wh}\left(\pi_{1} M\right)$ of $h$ is zero.

2. We now sketch the proof of (I) and (II). Important elements of it were announced in [7], [8]. An important role is played by Lees' recent classification theorem for topological immersions incodimension zero [11], and by Wall's surgery of nonsimply connected manifolds [15]; we suspect that one or both could be eliminated from the proof of (I), but they are essential in the proof of (II). In this regard see the weaker triangulation theorems of Lashof [9] and Lees [11] proved before (I).

HANDLE STRAIGHTENING PROBLEM $P(h)$. Consider a homeomorphism $h: B^{k} \times R^{n} \rightarrow V^{m}, k+n=m$, (where $B^{k}=$ standard $P L k$-ball in $R^{k}$ ) onto a $P L$ manifold $V$ so that $h \mid \partial B^{k} \times R^{n}$ is a PL homeomorphism. Can one find a topological isotopy $h_{t}: B^{k} \times R^{n} \rightarrow V^{n}, 0 \leqq t \leqq 1$, of $h=h_{0}$ such that

(1) $h_{1} \mid B^{k} \times B^{n}$ is $P L$,

(2) $h_{t}=h, 0 \leqq t \leqq 1$, on $\partial B^{k} \times R^{n} \cup B^{k} \times\left(R^{n}-r B^{n}\right)$ for some $r$ ?

- This makes good sense even when $M$ is noncompact, cf. [13, p. 74]. 
One should think of $B^{k} \times R^{n}$ as an open PL handle with core $B^{k} \times 0$. The analysis of $P(h)$ is based on the main diagram below, which for $k=0$, originated in [5].

\section{MAIN DIAGRAM}

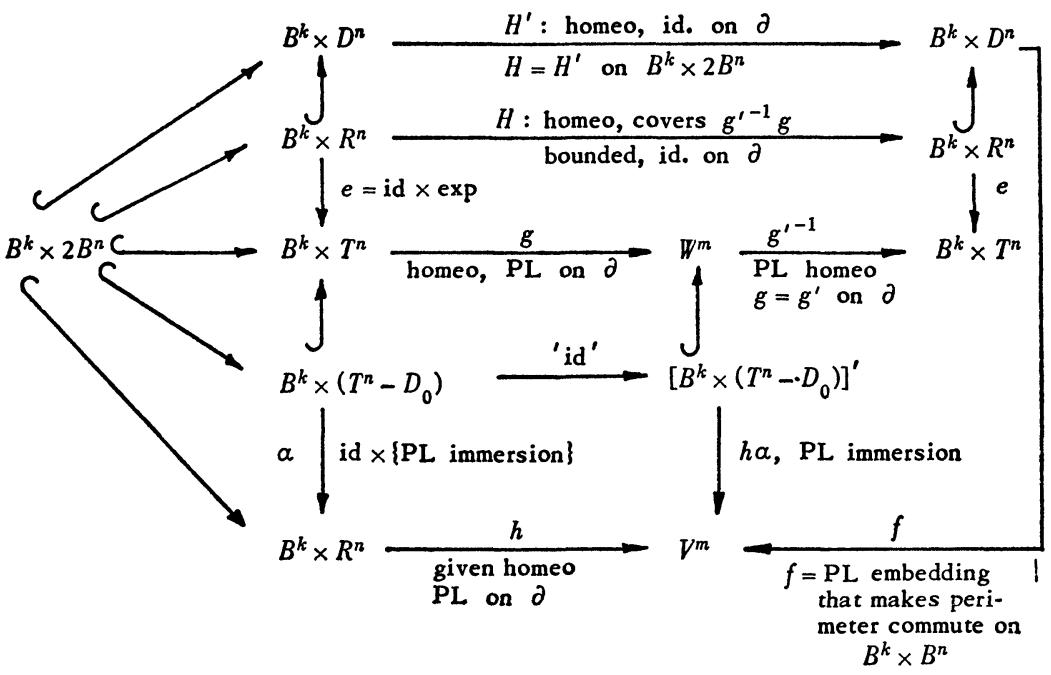

$m=n+k \geqq 5, \partial$ indicates boundary, $B^{k}=$ standard PL $k$-ball in $R^{k}$. $D^{n}=$ a PL $n$-ball with $\operatorname{int} D^{n}=R^{n}=$ euclidean $n$-space, so that bounded maps $R^{n} \rightarrow R^{n}$ extend by the identity to $D^{n}$.

$T^{n}=n$-torus, the $n$-fold product of circles; $D_{0}=$ a PL $n$-ball, collared in $T^{n}$.

Arrange that $h$ is PL near $\partial B^{k} \times R^{n}$ and successively construct $e, \alpha, W, g, g^{\prime}$ (when possible), $H, H^{\prime}, f$.

Explanations. $\alpha, e$ and the inclusions of $B^{k} \times 2 B^{n}$ are chosen so that the triangles at left commute; $B^{k} \times\left(T^{n}-D_{0}\right)$ with the unique PL structure making $h \alpha \mathrm{PL}$ is by definition $\left[B^{k} \times\left(T^{n}-D_{0}\right)\right]^{\prime} ; W$ is PL and "caps it off". $g$ extends $i d$. Finding $g$ ' is the

TORUS PROBLEM $Q(g)$. To extend $g \mid \partial B^{k} \times T^{n}$ to a $P L$ homeomorphism homotopic to $\mathrm{g}$.

Supposing $g^{\prime}$ solves $Q(g)$, form $H$ and 'squeeze' to allow extension to $H^{\prime}$; then define $f$ by engulfing to extend the natural PL identification $H^{\prime}\left(B^{k} \times B^{n}\right) \rightarrow h\left(B^{k} \times B^{n}\right)$. Since $f$ embeds the ball $B^{k} \times D^{n}$, for any isotopy $H_{t}^{\prime}$ of $H^{\prime}$ fixing $\partial$ (= boundary), there exists an isotopy $h_{t}$ of $h$ fixing $\partial$ and $B^{k} \times R^{n}-h^{-1} f\left(B^{k} \times D^{n}\right)$ so that, for each $t, h_{t}=f H_{t}^{\prime}$ on $B^{k} \times B^{n}$. Thus, if $H_{t}^{\prime}$ is the Alexander isotopy of $H^{\prime}$ to the identity, $h_{t}$ solves $P(h) !$ ! 
Proposition 1. If $g$ is derived from $h$ as above, $Q(g)$ is solvable $\Leftrightarrow P(h)$ is solvable.

Proof. It remains to show $\Longleftarrow$. Using a solution $h_{t}$ of $P(h)$ form an isotopy $\bar{h}: I \times B^{k} \times R^{n} \rightarrow I \times V$, fixing $\partial$, from $h$ to a PL homeomorphism. One can extend the construction of $g$ from $h$ to get from the isotopy $\bar{h}$ a homeomorphism $\bar{g}: I \times B^{k} \times T^{n} \rightarrow W^{\prime}$ with $\bar{g} \mid\{0\} \times B^{k} \times T^{n}$ $=g$, that is PL on $\partial$ minus $\{0\} \times$ int $B^{k} \times T^{n}$. Applying the $s$-cobordism theorem to $W^{\prime}$ one gets a solution of $Q(g)$.

Given the problem $P(h)$ consider the tangent bundle map $h_{*}: \tau\left(B^{k} \times R^{n}\right) \rightarrow \tau\left(V^{m}\right)$. Both bundles are PL trivial. Restricting over $B^{k} \times\{0\}$ in the source and projecting to the fiber in the target we get a map $\left(B^{k}, \partial B^{k}\right) \rightarrow\left(\mathrm{TOP}_{m}, \mathrm{PL}_{m}\right)$ (to be understood semisimplicially $[12, \S 2])$. Call its class $d(h) \in \pi_{k}\left(\mathrm{TOP}_{m}, \mathrm{PL}_{m}\right) \cong \pi_{k}\left(\mathrm{TOP}_{m} / \mathrm{PL}_{m}\right)$. Denote this group by $\Pi_{k}(m)$.

Proposition 2. $P(h)$ is solvable $\Leftrightarrow d(h)=0$.

Proof. $\Rightarrow$ is trivial. If $d(h)=0$, Lees' immersion theorem [11], together with its familiar PL counterpart due to Haefliger and Poenaru, provides a regular homotopy (modulo $\partial$ ) from $h$ to a PL immersion. The argument under Proposition 1 applies to solve $Q(g)$ and hence $P(h)$.

Proposition 2'. For each $x \in \Pi_{k}(m), k<m$, there exists a problem $P(h)$ with $d(h)=x$.

Proof. Immersion theory here provides an immersion $h_{0}: B^{k} \times R^{n}$ $\rightarrow R^{m}$, PL on $\partial$, such that if $V^{m}$ is $B^{k} \times R^{n}$ with the PL structure making $h_{0} \mathrm{PL}$, then $h=i d: B^{k} \times R^{n} \rightarrow V^{m}$ has $d(h)=x$.

For any solution $h_{t}$ of $P(h)$, the induced tangent bundle map gives a map $f: I \times B^{k} \rightarrow \mathrm{TOP}_{m}$ sending $I \times \partial B^{k} \cup\{1\} \times B^{k}$ into $\mathrm{PL}_{m}$. Thus, given two solutions $h_{t}, h_{t}^{\prime}$ of $P(h)$ we can piece together $f, f^{\prime}$ to get a 'difference' class $\delta\left(h_{t}, h_{t}^{\prime}\right) \in \pi_{k+1}\left(\mathrm{TOP}_{m}, \mathrm{PL}_{m}\right)=\pi_{R+1}(m)$.

Proposition 3. Let $h_{t}$ solve $P(h)$ for $h: B^{k} \times R^{n} \rightarrow V^{m}, k<m$. Given any $y$ in $\Pi_{k+1}(m+1)$ there exists a solution $h_{t}^{\prime}$ of $P(h)$ such that $s \delta\left(h_{t}, h_{t}^{\prime}\right)$ $=y$ ( $s$ denotes stabilization which by (II) is an isomorphism).

INDICATION OF PROOF. One can reduce to the special case where $h_{t}$ is the identity solution of $P\left(i d \mid B^{k} \times R^{n}\right)$. Proposition $2^{\prime}$ provides a problem $P\left(h_{1}\right), h_{1}: B^{k+1} \times R^{n} \rightarrow V^{m+1}$ with $d\left(h_{1}\right)=y$, which yields a torus problem $Q\left(g_{1}\right), g_{1}: B^{k+1} \times T^{n} \rightarrow W^{m+1}$. Impose on $B^{k+1} \times T^{n}$ $=I \times B^{k} \times T^{n}$ the PL structure $\Sigma$ making $g_{1}$ PL. $\Sigma$ is standard on $\partial$. Apply the $s$-cobordism theorem to $(I ; 0,1) \times B^{k} \times T^{n}$ with structure 
$\Sigma$ to derive a PL automorphism $\theta$ of $\{1\} \times B^{k} \times T^{n}$ that fixes $\partial$. Solve $P\left(i d \mid B^{k} \times R^{n}\right)$ using $\theta$ (for $g^{\prime}$ of the main diagram) in place of $i d$, and call the solution $h_{t}^{\prime}$. Then $s \delta\left(i d, h_{t}^{\prime}\right)=y$.

Proof of (II). (a) For $m>k>3$ there is an elementary proof that $\Pi_{k}(m)=0$. It is an induction on $n=m-k \geqq 1$ exploiting the main diagram. Also, surgery can be used as in $(b)$.

(b) $\Pi_{k}(m)=0$ for $m \geqq 5$ and $k=0,1,2$. Represent $x \in \Pi_{k}(m)$ by a problem $P(h)$ and pass to the torus problem $Q(g)$. There is an obstruction $[g] \in H^{3-k}\left(T^{n} ; Z_{2}\right)$ to solving $Q(g)$. For $[g]$ to exist, $g$ need only be a homotopy equivalence which is a PL homeomorphism on $\partial$, and, strictly speaking, $[g]$ is the obstruction to homotoping $g$ (modulo $\partial$ ) to a PL homeomorphism. Note that to solve $P(h)$ it suffices to solve $Q(\bar{g})$ where $\bar{g}$ is the covering of $g$ for $(2 Z)^{n} \subset Z^{n}$ $=\pi_{1}\left(T^{n}\right)$.

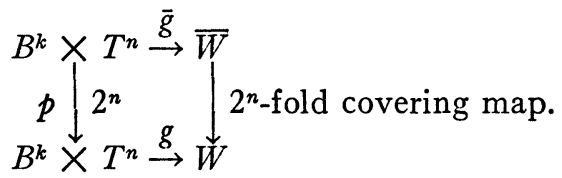

To see this just add a new tier over $g$ in the main diagram! Now $[\bar{g}]=p^{*}[g]$. But $p^{*}$ kills $H^{3-k}\left(T^{n} ; Z_{2}\right)$. Thus $P(h)$ is solvable and $x=0$. (Also $Q(g)$ is solvable by Proposition 1 , so $[g]$ was already $0 !$ )

(c) $\Pi_{3}(m) \subset Z_{2}$ for $m \geqq 5$. In the above argument $p^{*}=i d$. But by inspection of definitions $x \mapsto[g] \in H^{0}\left(T^{n} ; Z_{2}\right)$ is additive.

(d) Stability: $s: \Pi_{3}(m) \rightarrow \Pi_{3}(m+1)$ is an isomorphis $m, m \geqq 5$. That $s$ is onto follows by the descent argument for (a). To prove $s$ is injective we check that, if $P(h)$ gives $Q(g)$ with surgery obstruction $y \in Z_{2}$, then $P\left(h \times 1_{R}\right)$ also gives $y \in Z_{2}$.

Prior to this work, C. T. C. Wall, W. C. Hsiang, and J. Shaneson (jointly), and A. Casson understood the classification of homotopy tori (the case when $k=0$ above). ${ }^{5}$ When our specific questions (see [6] and [8]) were posed, Wall [16] and Hsiang and Shaneson [4], independently verified that $[\bar{g}]=p^{*}[g]$ and extended their work to the cases $k \neq 0$. It seems to us that the fibration theorem of Farrell [1] reworked in [2] and [15]) plays an essential role in the construction and use of $[\mathrm{g}]$. We thank W. Browder for a very transparent definition of $[g]$.

Propositions $1,2,2^{\prime}, 3$ and the above stability permit us to prove (I).

Uniqueness (Hauptvermutung). Consider an unbounded TOP manifold $M$ with two PL structures $\Sigma, \Sigma^{\prime}$ giving reductions $\rho, \rho^{\prime}$ of

- Casson apparently had examined the general case $k \geqq 0$ [16]. 
$\tau(M)$ to PL microbundle that are (stably) related by a deformation $\sigma$ of reductions. Take a handle decomposition of $M$, and suppose that, for a subhandlebody $M_{0}$, one has found an isotopy $h_{t}, 0 \leqq t \leqq s$, of $1_{M}$ with $h_{s} \mid M_{0}$ being PL, which realizes $\sigma \mid M_{0}$. If $H$ is a $k$-handle attached to $M_{0}$, the obstruction in $\pi_{k}$ (TOP/PL) (Proposition 2) to prolonging $h_{t}$ fixing $M_{0}$ to an isotopy $h_{t}, 0 \leqq t \leqq s+1$, with $h_{s+1} \mid M_{0} \cup H$ being $\mathrm{PL}$, can be identified with the obstruction to extending over $M_{0} \cup H$ the deformation determined by $h_{t} \mid M_{0}$ between the reductions given by $\Sigma$ and $\Sigma^{\prime}$. It is zero because $\sigma \mid M_{0} \cup H$ exists. Proposition 3 allows us to choose $h_{t}, 0 \leqq t \leqq s+1$, so as to realize $\sigma \mid M_{0} \cup H$. This indicates how to construct inductively the desired isotopy. By using small handles, one can make this isotopy small. In a relative form, which this argument also yields, the case for open manifolds takes care of manifolds with boundary.

Existence (Triangulation). Once it is stated in a relative form (see [7]) we can assume that $\partial M=\varnothing$ and (by passing to charts of a locally finite cover) that $M$ is triangulable. Again we use a handle induction, and spread the PL structure handle by handle. Proposition $2^{\prime}$ takes the above role of Proposition 3, so this argument for existence is simpler than that for uniqueness above.

Added IN Proof. We have proved that $\pi_{3}$ (TOP/PL) is $Z_{2}$ not 0 , see [17]. Here is an argument in outline. Surgery provides a homotopy equivalence $g: B^{1} \times T^{n} \rightarrow W, n \geqq 5$, that is a PL homeomorphism on $\partial$ and has nonzero invariant $[g] \in H^{2}\left(T^{n} ; Z_{2}\right.$ ). (Here and below compare proof of II.) Using the $s$-cobordism theorem (cf. Proposition 3) one derives from $g$ a PL automorphism $h$ of $T^{n}$, well-defined by $[g]$ up to PL pseudo-isotopy (concordance). Let $g_{\lambda}, \lambda=1,3,5, \cdots$ be the standard $\lambda^{n}$-fold covering of $g$ and let $h_{\lambda}$ be a $\lambda^{n}$-fold covering of $h$ derived from $g_{\lambda}$. For $\lambda$ large, $h_{\lambda}$ can be arbitrarily close to $i d \mid T^{n}$; hence is topologically isotopic to $i d \mid T^{n}$ by a result of Cernavskii (Doklady 1968) provable [6] by a method of [5]. Thus if $\pi_{*}(\mathrm{TOP} / \mathrm{PL})$ were zero, $h_{\lambda}$ would be at least PL pseudo-isotopic to $i d \mid T^{n}$. But it is not, since $[g \lambda]=[g] \neq 0$ as $\lambda$ is odd. Therefore $\pi_{3}(\mathrm{TOP} / \mathrm{PL})=Z_{2}$.

This discovery leads to many striking conclusions (e.g. see Notices Amer. Math. Soc., June 1969). These will be discussed fully in a paper devoted to a careful development of our results.

\section{REFERENCES}

1. F. T. Farrell, Thesis, Yale University, New Haven, Conn., 1967.

2. F. T. Farrell and W. C. Hsiang, Manifolds with $\pi_{1}=Z \times{ }_{\alpha} G$ (to appear).

3. M. W. Hirsch, On non-linear cell-bundles, Ann. of Math (2) 84 (1966), 373-385. 
4. W. C. Hsiang and J. L. Shaneson, Fake tori, the annulus conjecture and the conjectures of Kirby, Proc. Nat. Acad. Sci. U.S.A. (to appear).

5. R. C. Kirby, Stable homeomorphisms and the annulus conjecture, Ann. of Math. (2) 90 (1969).

6. - Announcement distributed with preprint of [5], 1968.

7. R. C. Kirby and L. C. Siebenmann, $A$ triangulation theorem, Notices Amer. Math. Soc. 16 (1969), 433.

8. R. C. Kirby, L. C. Siebenmann and C. T. C. Wall, The annulus conjecture and triangulation, Notices Amer. Math. Soc. 16 (1969), 432.

9. R. K. Lashof, Lees' immersion theorem and the triangulation of manifolds, Bull. Amer. Math. Soc. 75 (1969), 535-538.

10. R. K. Lashof and M. Rothenberg, Triangulation of manifolds. I, II, Bull. Amer. Math. Soc. 75 (1969), 750-754, 755-757.

11. J. A. Lees, Immersions and surgeries of topological manifolds, Bull. Amer. Math. Soc. 75 (1969), 529-534.

12. C. P. Rourke and B. J. Sanderson, On the homotopy theory of $\Delta$-sets (to appear).

13. L. C. Siebenmann, On the homotopy type of compact topological manifolds, Bull. Amer. Math. Soc. 74 (1968), 738-742.

14. D. P. Sullivan, On the hauptvermutung for manifolds, Bull. Amer. Math. Soc. 63 (1967), 598-600.

15. C. T. C. Wall, Surgery on compact manifolds, preprint, University of Liverpool.

16. - On homotopy tori and the annulus theorem, Proc. London Math. Soc. (to appear).

17. R. C. Kirby and L. C. Siebenmann, For manifolds the Hauptvermutung and the triangulation conjecture are false, Notices Amer. Math. Soc. 16 (1969), 695.

University of California, Los Angeles, California 90024 and Institute for Advanced Study, Princeton, New Jersey 08540 